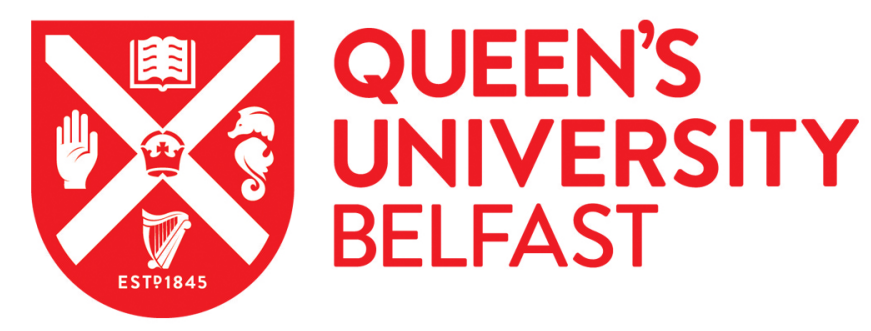

\title{
On the Aperture Efficiency of Intelligent Reflecting Surfaces
}

Chou, S-K., Yurduseven, O., Ngo, H-Q., \& Matthaiou, M. (2020). On the Aperture Efficiency of Intelligent Reflecting Surfaces. IEEE Wireless Communications Letters. https://doi.org/10.1109/LWC.2020.3039483

\author{
Published in: \\ IEEE Wireless Communications Letters
}

\section{Document Version:}

Peer reviewed version

Queen's University Belfast - Research Portal:

Link to publication record in Queen's University Belfast Research Portal

Publisher rights

Copyright 2020 IEEE. This work is made available online in accordance with the publisher's policies. Please refer to any applicable terms of use of the publisher.

\section{General rights}

Copyright for the publications made accessible via the Queen's University Belfast Research Portal is retained by the author(s) and / or other copyright owners and it is a condition of accessing these publications that users recognise and abide by the legal requirements associated with these rights.

Take down policy

The Research Portal is Queen's institutional repository that provides access to Queen's research output. Every effort has been made to ensure that content in the Research Portal does not infringe any person's rights, or applicable UK laws. If you discover content in the Research Portal that you believe breaches copyright or violates any law, please contact openaccess@qub.ac.uk. 


\title{
On the Aperture Efficiency of Intelligent Reflecting Surfaces
}

\author{
Shih-Kai Chou, Student Member, IEEE, Okan Yurduseven, Senior Member, IEEE, Hien Quoc Ngo, Senior \\ Member, IEEE, and Michail Matthaiou, Senior Member, IEEE
}

\begin{abstract}
The concept of intelligent reflecting surfaces (IRS) has recently gained significant attention due to their ability to manipulate the impinging electromagnetic signals and offer anomalous reflections. In this letter, we derive and address the missing piece of prior works, namely the IRS aperture efficiency. Our work provides practical guidelines for selecting the aperture size of the reflecting surface from an antenna designing point of view by taking tapering level into account, and also, revisits the pathloss expression based on a realistic physical model.
\end{abstract}

Index Terms-Aperture efficiency, intelligent reflecting surfaces, pathloss model.

\section{INTRODUCTION}

A $\mathrm{S}$ we are witnessing the steady roll-out of the fifthgeneration $(5 \mathrm{G})$ cellular networks, the importance of massive multiple-input multiple-output (M-MIMO), becomes unquestionable. Looking ahead, a number of technologies have been proposed to extend the capabilities of M-MIMO, namely cell-free M-MIMO [1], millimeter wave (mmWave) communications [2]. These concepts are theoretically attractive, yet, they entail fundamental technological challenges, such as the increased cost of RF electronics at higher frequencies along with the short operating range, as well as, the excessive amount of signalling overhead requirements in cell-free M-MIMO to name but a few. The above discussion reveals that a technology which can harness recent advances in electromagnetics, communications and computing by maintaining the signal processing and hardware complexity at affordable levels is of pivotal importance. The focus of this paper is on such a technology, namely, intelligent reflecting surfaces (IRS), whose purpose is to perform operations such as reflect, refract, absorb, polarisation adjustment, and data modulation in order to guarantee high quality data delivery [3]. The potential of such a solution is outstanding, and it has recently come at the forefront of wireless communications research; not surprisingly, it is considered as one of the most promising technologies for the sixth generation $(6 \mathrm{G})$ communication systems [4]: these reconfigurable metasurfaces can be controlled either locally or at network level in order to customize the propagation of the radio waves, thereby

The authors are with the Institute of Electronics, Communications and Information Technology (ECIT), Queen's University Belfast, Belfast, U.K., (email: \{schou01, okan.yurduseven, hien.ngo,m.matthaiou\}@qub.ac.uk)

The work of O. Yurduseven was in part supported by a research grant from the Leverhulme under Research Leadership Award RL-2019-019. The work of H. Q. Ngo was supported by the UK Research and Innovation Future Leaders Fellowships under Grant MR/S017666/1. The work of M. Matthaiou was supported by EPSRC, UK, under grant EP/P000673/1. improving the network coverage through low-energy and lowcomplexity sensing [5], and basic mathematical operations [6]. This topological approach can offer seamless cellular coverage, as well as, surveillance and localization. The ability of enhancing the energy transmission efficiency and range with the large aperture of IRS has motivated diverse applications, namely simultaneous wireless information and power transfer (SWIPT) [7] and non-orthogonal multiple access (NOMA) [8] by compensating the significant power loss over long distance via passive beamforming. Furthermore, the physical layer security can be optimized when the active transmit beamforming and passive reflect beamforming at the IRS are jointly designed [9].

The common characteristic of the biggest body of IRS literature is that they address the problem of system modeling using idealistic information theoretic tools by often ignoring inherent electromagnetic phenomena. An illustrative example is [10, Eq. (9)], which proposes that the field strength of an IRS scales quadratically with the number of reflecting plates. However, this approach ignores the presence of phase errors and extra losses on an IRS (e.g., spillover loss from the missing part of the reference wave that is not captured on the aperture, conduction loss, dielectric loss etc). In this paper, we are moving away from the state-of-the-art, such as [7]-[9], [11], [12], and inform the theoretical modeling of IRSbased communication by starting from basic electromagnetic principles. The specific paper contributions are the following:

- We consider a rectangular IRS and provide both numerical and analytical approaches of a key factor in the IRS communication system, namely, aperture efficiency, which previous literature did not put into consideration.

- We provide a physical-based pathloss expression of the signal that traverses the path from the source and relayed by the IRS to the destination. Our model is an amelioration of previous results and enables to pursue a rigorous performance characterization of future IRS topologies.

- We investigate the size of IRS based from an antenna designing point of view, when we take the aperture efficiency into account, and demonstrate that there is a trade off between the distance from the source to IRS and the aperture size.

\section{EFFICIENCY OF RECTANGULAR IRS}

Ideally, the maximum achievable gain for a reflecting antenna array is obtained by assuming that the receiving amplitude and phase are uniformly distributed and that there is 
no spillover and taper losses at the same time. Mathematically speaking, it can be denoted as $G_{\max }$ and defined as [13]

$$
G_{\max }=\frac{4 \pi}{\lambda^{2}} A_{\mathrm{p}}
$$

where $\lambda$ and $A_{p}$ are the wavelength of the incident waves and the aperture size of the reflective antenna array. However, in practice, an aperture antenna of finite size has an effective gain that is defined as

$$
G_{\mathrm{eff}}=\varepsilon_{\mathrm{ap}} G_{\max }
$$

where $\varepsilon_{\text {ap }}$ is the aperture efficiency, that is dependent on several factors, such as, the radiation pattern of the feeding antenna (or source), the excitation footprint of the source radiation pattern across the reflecting surface, the position of the source with respect to the reflecting surface and the operating frequency. The aperture efficiency of a reflecting surface can be defined as [13]

$$
\varepsilon_{\mathrm{ap}} \triangleq \varepsilon_{\mathrm{cd}} \varepsilon_{\mathrm{s}} \varepsilon_{\mathrm{t}} \varepsilon_{\mathrm{p}} \varepsilon_{\mathrm{b}}
$$

in which $\varepsilon_{\mathrm{cd}}$ is defined as the radiation efficiency (governed by intrinsic material losses, such as dielectric and conduction losses), $\varepsilon_{\mathrm{p}}$ as phase efficiency and $\varepsilon_{\mathrm{b}}$ is the blockage efficiency, respectively. In this paper, we ignore the intrinsic material losses and assume a point source to illuminate the IRS aperture, the aperture efficiency of a reflecting surface is mainly dominated by spillover efficiency $\left(\varepsilon_{\mathrm{S}}\right)$ and taper efficiency $\left(\varepsilon_{\mathrm{t}}\right)$ and the rest of efficiencies are assumed to be ideal (equal to 1) [14]. Thus, the aperture efficiency can be rewritten as

$$
\varepsilon_{\mathrm{ap}}=\varepsilon_{\mathrm{s}} \varepsilon_{\mathrm{t}},
$$

and since $\varepsilon_{\text {ap }}$ is a real number equal to or less than 1 , in real-world scenarios, we have $G_{\text {eff }} \leq G_{\max }$.

Note that in the past literature of IRS (e.g. [10], [15] among others), the aperture efficiency is unrealistically assumed to be 1 , which means all the radiation from the source antenna is perfectly captured by the reflecting surface and the received waves have uniform distribution of amplitudes.

\section{A. Spillover and Taper Efficiency}

Spillover efficiency measures the amount of radiation from the source antenna that is reflected by the reflective antenna array. Because of the finite size of the array, some of the radiation from the source antenna will deviate from the main axis and eventually not being reflected. According to the definition of spillover efficiency, we have

$$
\varepsilon_{\mathrm{s}}=\frac{\iint_{S}\left|\overrightarrow{E_{\mathrm{F}}}\right|^{2} d S}{\iint_{S^{\prime}}\left|\overrightarrow{E_{\mathrm{F}}}\right|^{2} d S^{\prime}} \triangleq \frac{I_{1}}{I_{2}},
$$

where $S$ and $S^{\prime}$ denote the surface of the IRS and the surface of the hemisphere in front of the source (see Fig. 1), while $\overrightarrow{E_{\mathrm{F}}}$ is the electric field intensity of the source antenna. On the other hand, the taper efficiency (also known as illumination efficiency) is the representation of how uniform the electrical field is across the surface of reflective surface. In practice, the aperture fields will tend to diminish away from the main axis of the reflector, which leads to lower gain, and this loss is captured by this parameter. Therefore, we can define the taper efficiency as

$$
\varepsilon_{\mathrm{t}}=\frac{1}{A_{\mathrm{p}}} \frac{\left|\iint_{S^{\prime \prime}} \overrightarrow{E_{\mathrm{F}}} \cdot \overrightarrow{E_{\text {element }}} d S^{\prime \prime}\right|^{2}}{\iint_{S}\left|\overrightarrow{E_{\mathrm{F}}}\right|^{2} d S},
$$

where $S^{\prime \prime}$ is the surface of the sphere limited by the reflecting antenna array aperture, $\vec{E}_{\text {element }}$ is the electrical field, which is reflected by the element on the IRS, and - is the inner product of two vectors.

\section{B. Derivation of Spillover and Taper Efficiency}

We can easily see that the denominator of (5) is the same as the nominator of (4). Now, we define $I_{3}$ as

$$
I_{3} \triangleq\left|\iint_{S^{\prime \prime}} \overrightarrow{E_{\mathrm{F}}} \cdot \vec{E}_{\text {element }} d S^{\prime \prime}\right|,
$$

therefore, we can have the taper efficiency rewritten as

$$
\varepsilon_{\mathrm{t}}=\frac{1}{A_{\mathrm{p}}} \frac{I_{3}^{2}}{I_{1}} .
$$

We now consider the radiation beam of the source antenna, which has the power pattern $\left(U_{\mathrm{F}}(\theta, \phi)\right)$, and it is modeled as

$$
U_{\mathrm{F}}(\theta, \phi)=E_{0} \cos ^{q_{\mathrm{F}}}(\theta),
$$

where $E_{0}$ is the magnitude of the electrical field and $q_{\mathrm{F}}$ is the parameter determining the directivity of the source antenna and the shape of the pattern: as $q_{\mathrm{F}}$ increases, the beam becomes narrower and delivers higher directivity, while $\theta$ and $\phi$ represent the elevation angle and azimuth angle. The Poynting vector of the source defined by the power pattern (8) can be written in terms of the source region spherical coordinates as

$$
\overrightarrow{U_{\mathrm{F}}}(\hat{r})=\frac{E_{0}}{R} \cos ^{q_{\mathrm{F}}}(\theta) \hat{r},
$$

where $\hat{r}$ is the unit vector from the source antenna to the elements on the IRS, while $R$ is the distance from the source antenna to the surface of the IRS. We assume that the incident wave from the source antenna is travelling along the $z$ direction and polarized along the $y$ axis. Substituting (9) into the electrical field intensity, we have $\overrightarrow{E_{\mathrm{F}}}$ in spherical coordinates as

$$
\overrightarrow{E_{\mathrm{F}}}=\frac{E_{0}}{R} \cos ^{q_{\mathrm{F}}}(\theta) \sin (\phi) \cdot \hat{i}_{\theta},
$$

where $\hat{i}_{\theta}$ is the unit position vector from the source to IRS. As a result, $I_{1}, I_{2}$ and $I_{3}$ can be rewritten as

$$
\begin{aligned}
& I_{1}=\iint_{S}\left|\frac{E_{0} \cos ^{q_{\mathrm{F}}}(\theta)}{R} \sin (\phi)\right|^{2} d S, \\
& I_{2}=\iint_{S^{\prime}}\left|\frac{E_{0} \cos ^{q_{\mathrm{F}}}(\theta)}{R} \sin (\phi)\right|^{2} d S^{\prime}, \\
& I_{3}=\iint_{S}\left|\frac{E_{0} \cos ^{q_{\mathrm{F}}+q_{\mathrm{R}}+1}(\theta)}{R} \sin (\phi)\right| d S,
\end{aligned}
$$




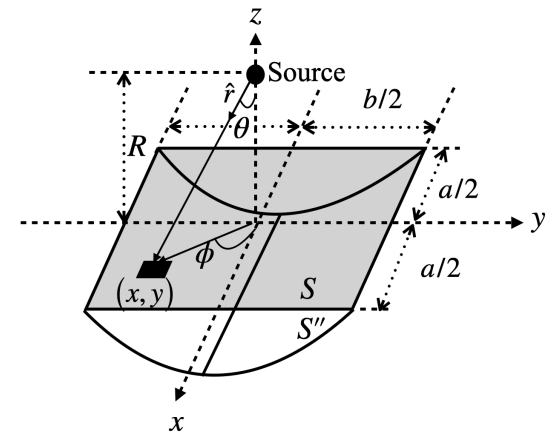

Fig. 1: Coordinate system and configuration parameters of a rectangular IRS.

where $q_{\mathrm{R}}$ is defined as the directivity of the reflective antenna array. Figure 1 shows the rectangular coordinates system used to determine the efficiencies of the rectangular IRS. Since we consider a reflecting surface with the aperture area shaped as rectangular, it is easier to model $I_{1}, I_{2}$ and $I_{3}$ in a Cartesian coordinate system; thus, we can express $\cos (\theta)$ and $\sin (\phi)$ in Cartesian coordinates according to

$$
\cos (\theta)=\frac{R}{\sqrt{R^{2}+x^{2}+y^{2}}}, \quad \sin (\phi)=\frac{y}{\sqrt{x^{2}+y^{2}}} .
$$

By substituting $\cos (\theta)$ and $\sin (\phi)$, we can have

$$
\begin{gathered}
I_{1}=\frac{E_{0}^{2}}{R^{2}} \int_{\frac{-a}{2}}^{\frac{a}{2}} \int_{\frac{-b}{2}}^{\frac{b}{2}} R^{2 q_{\mathrm{F}}+3} y^{2}\left(R^{2}+x^{2}+y^{2}\right)^{-\left(q_{\mathrm{F}}+\frac{3}{2}\right)} \\
\times\left(x^{2}+y^{2}\right)^{-1} d x d y
\end{gathered}
$$

and

$$
\begin{aligned}
I_{3}=\int_{-\frac{a}{2}}^{\frac{a}{2}} \int_{-\frac{b}{2}}^{\frac{b}{2}} & \frac{E_{0}}{\sqrt{R^{2}+x^{2}+y^{2}}}\left(\frac{R}{\sqrt{R^{2}+x^{2}+y^{2}}}\right)^{q_{\mathrm{F}}+q_{\mathrm{R}}+1} \\
& \times\left(\frac{y}{\sqrt{x^{2}+y^{2}}}\right)^{2} d x d y
\end{aligned}
$$

where $a$ and $b$ are the lengths of IRS in the $x$ and $y$ direction, respectively, while $I_{2}$ can be derived analytically as follows:

$$
\begin{aligned}
I_{2} & =\int_{\theta=0}^{\frac{\pi}{2}} \int_{\phi=0}^{2 \pi} \frac{E_{0}^{2}}{R^{2}} \cos ^{2 q_{\mathrm{F}}}(\theta) \sin ^{2}(\phi) R^{2} \sin (\theta) d \theta d \phi \\
& =E_{0}^{2} \pi\left(\frac{1}{2 q_{\mathrm{F}}+1}\right), \mathbb{R}\left\{q_{\mathrm{F}}\right\}>-\frac{1}{2} .
\end{aligned}
$$

Unfortunately, $I_{1}$ and $I_{3}$ do not admit an analytical solution due to functions that are powered by $-\left(q_{\mathrm{F}}+\frac{3}{2}\right)$ and $q_{\mathrm{F}}+q_{\mathrm{R}}+$ 1. Therefore, we now introduce two approximation methods, which are Gaussian-Legendre Quadrature Rule (GLQR) and Taylor Expansion to numerically evaluate these integrals.

1) Gaussian-Legendre Quadrature Rule (GLQR): The reason we use GLQR is that it is generally more accurate than the Newton-Cotes quadrature technique.
Lemma 1: By using GLQR, we can obtain a numerical solution for $I_{1}$

$$
\begin{gathered}
I_{1} \approx \frac{a b E_{0}^{2} R^{2 q_{\mathrm{F}}+1}}{4} \sum_{i=1}^{n} \sum_{j=1}^{m}\left[\left(\frac{a \xi_{i}}{2}\right)^{2}+\left(\frac{b \eta_{j}}{2}\right)^{2}\right]^{-1} \\
\times\left[R^{2}+\left(\frac{a \xi_{i}}{2}\right)^{2}+\left(\frac{b \eta_{j}}{2}\right)^{2}\right]^{-q_{E}-\frac{3}{2}}\left(\frac{b \eta_{j}}{2}\right)^{2} w_{1, i} w_{2, j},
\end{gathered}
$$

where $n$ and $m$ are the number of sample points, and $\left\{\left(\xi_{i}, \eta_{i}\right) \mid-1 \leq \xi_{i} \leq 1,-1 \leq \eta_{i} \leq 1\right\}$, while $w_{1, i}$ and $w_{2, j}$ are Gaussian-Legendre weighting functions:

$$
w_{1, i}=\frac{2}{\left(1-x_{i}^{2}\right)\left[P_{n}^{\prime}\left(x_{i}\right)\right]^{2}}, w_{2, j}=\frac{2}{\left(1-y_{j}^{2}\right)\left[P_{m}^{\prime}\left(y_{j}\right)\right]^{2}},
$$

where $x_{i}$ and $y_{j}$ are the roots of the Legendre polynomial $P_{n}(\cdot)$.

Lemma 2: By using GLQR, we can obtain a numerical solution for $I_{3}$ as

$$
\begin{gathered}
I_{3} \approx \frac{E_{0}^{2} R^{q_{\mathrm{F}}+q_{\mathrm{R}}+1} a b}{4} \sum_{i=1}^{n} \sum_{j=1}^{m}\left[\left(\frac{a \xi_{i}}{2}\right)^{2}+\left(\frac{b \eta_{j}}{2}\right)^{2}\right]^{-1} \\
\times\left[R^{2}+\left(\frac{a \xi_{i}}{2}\right)^{2}+\left(\frac{b \eta_{j}}{2}\right)^{2}\right]^{\frac{-q_{\mathrm{F}}-q_{\mathrm{R}}-2}{2}}\left(\frac{b \eta_{j}}{2}\right)^{2} w_{1, i} w_{2, j} .
\end{gathered}
$$

2) Taylor Expansion: We can approximate the integration problem (15) and (16) with Taylor expansion and further turn them into tractable closed-forms. We can observe from (15) and (16), that the term $R^{2}+x^{2}+y^{2}$ can be rewritten as

$$
\left(R^{2}+x^{2}+y^{2}\right)=R^{2}\left(1+\frac{x^{2}+y^{2}}{R^{2}}\right) .
$$

By defining $z_{\mathrm{s}}$ as

$$
z_{\mathrm{s}}=\frac{x^{2}+y^{2}}{R^{2}}, \quad\left|z_{\mathrm{s}}\right| \leq 1,
$$

and by utilizing the property of Taylor expansion $\left(\frac{1}{1+z}\right)^{p}=$ $\sum_{l=0}^{\infty} C_{l} z^{l}$, we can obtain the closed form of (15) and (16), by a third-order Taylor expansion:

$$
\begin{aligned}
I_{1} \approx & R^{-2}\left[\left(\frac{a^{2}+b^{2}}{2}\right) \tan ^{-1}\left(\frac{a}{b}\right)-\frac{q_{\mathrm{F}}+\frac{3}{2}}{3} a b^{3}+\frac{a b}{2}\right] \\
& +R^{-4}\left[\frac{\left(q_{\mathrm{F}}+\frac{3}{2}\right)\left(q_{\mathrm{F}}+\frac{1}{2}\right) a^{2} b^{3}}{144}+\frac{a b^{5}}{160}\right], \\
I_{3} \approx & R^{-1}\left[\left(\frac{a^{2}+b^{2}}{2}\right) \tan ^{-1}\left(\frac{a}{b}\right)+\frac{a b}{2}\right]+R^{-3} a b^{3} \\
& \times \frac{\left(q_{\mathrm{F}}+q_{\mathrm{R}}+2\right)}{24}\left[\frac{\left(q_{\mathrm{F}}+q_{\mathrm{R}}+4\right)\left(5 a^{2}+9 b^{2}\right)}{240}-1\right] .
\end{aligned}
$$

By observing (20) and (21), several parameters can be fine tuned to improve the aperture efficiency: for example, when 
the distance between the source and the IRS is large and the IRS diameter is fixed, we can adjust the directivity of the source antennas to increase the aperture efficiency of the IRS.

\section{PATHLOSS MODELING}

When it comes to the pathloss in free space and within the far-field regime, we naturally consider the Friis formula

$$
P_{\mathrm{R}}=\frac{P_{\mathrm{S}} G_{\mathrm{S}} G_{\mathrm{R}} \lambda^{2}}{\left(4 \pi R_{\mathrm{S}, \mathrm{IRS}}\right)^{2}}
$$

where $P_{\mathrm{S}}$ and $G_{\mathrm{S}}$ are the power and gain of the source antenna respectively, while $P_{\mathrm{R}}$ and $G_{\mathrm{R}}$ are the power and gain of the IRS and $R_{\mathrm{S}, \mathrm{IRS}}$ is the distance from the source to the IRS. Here, we consider line-of-sight ( $\mathrm{LoS})$ setup, since it is reasonable for systems deployed in indoor or outdoor open spaces such as rural areas, and for mmWave wireless systems with small cell sizes.

Recall now the relationship between the reflecting array antenna gain and efficiency; we can define $G_{\mathrm{eff}}$ as $G_{\mathrm{R}}$ and substitute (1) and (2) into (22); then, we arrive at

$$
\frac{P_{\mathrm{R}}}{P_{\mathrm{S}}}=\frac{G_{\mathrm{S}} \varepsilon_{\mathrm{s}} \varepsilon_{\mathrm{t}} a b}{4 \pi R_{\mathrm{S}, \mathrm{IRS}}^{2}} .
$$

Unfortunately, (23) does not consider the fact that the effective aperture size changes as we steer the radiation from the optical axis such that the decay of the effective aperture size can be approximated by a cosine pattern. Thus, (23) can be rewritten by considering the direction of the incident beam [16]. The receiving gain of the IRS then becomes

$$
G_{\mathrm{R}, \text { beam }}=G_{\mathrm{R}} \cos \theta_{\text {axis }}, \quad 0 \leq \theta_{\text {axis }} \leq \frac{\pi}{2} .
$$

By substituting (24) into (22) we can have

$$
\frac{P_{\mathrm{R}}}{P_{\mathrm{S}}}=\frac{G_{\mathrm{S}} \varepsilon_{\mathrm{s}} \varepsilon_{\mathrm{t}} a b \cos \theta_{\mathrm{axis}}}{4 \pi R_{\mathrm{S}, \mathrm{IRS}}^{2}} .
$$

As a result, the pathloss expression from source to destination through the reflecting IRS can be determined as

$$
\beta_{\mathrm{IRS}}=\frac{G_{\mathrm{S}} G_{\mathrm{D}}}{(4 \pi)^{2}}\left(\frac{a b}{R_{\mathrm{S}, \mathrm{IRS}} R_{\mathrm{IRS}, \mathrm{D}}}\right)^{2} \varepsilon_{\mathrm{ap}}^{2} \cos ^{2}\left(\theta_{\text {axis }}\right),
$$

where $G_{\mathrm{D}}$ is the gain of the receiving antenna, and $R_{\mathrm{IRS}, \mathrm{D}}$ is the distance between the IRS and the destination. As we can see in (26), when we consider $\beta_{\text {IRS }}$ as a function of $\theta_{\text {axis }}$, it has peak value at $\theta_{\mathrm{IRS}}=0$. This implies that the pathloss expression from the source to the IRS becomes maximum when the source is located on the optical axis.

\section{NUMERICAL RESULTS}

In this section, we present simulation results to validate our theoretical derivation of the aperture efficiency and our pathloss expression. The comparison of GLQR and Taylor expansion method is shown in Fig. 2, where we can see the behavior of spillover, taper and aperture efficiencies with the diameter equal to $10 \lambda$ and the frequency is $3 \mathrm{GHz}$, whist the directivities are set as $q_{\mathrm{F}}=9$ and $q_{\mathrm{R}}=1$ [13]. We note that in Section II, we provided physical insights into the aperture efficiency definition in terms of the source radiated

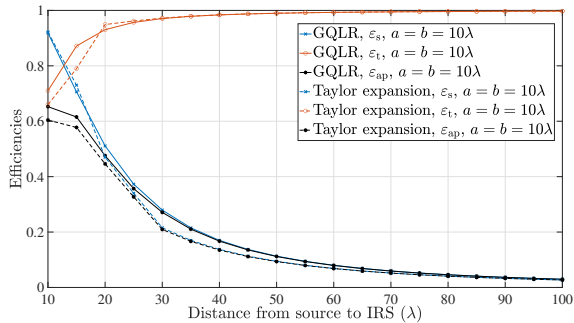

Fig. 2: Spillover, taper and aperture efficiency performance.

fields projected onto the IRS aperture, thus, the result is valid regardless of the radiation zone.

As we can see from (26), the diameter of IRS is also an important parameter for calculating $\beta_{\text {IRS }}$. However, we cannot have an infinitely big aperture to capture all the signal power while satisfying the far-field constraint. Hence, we now consider a more practical scenario and provide a cost-effective way to define the size of the IRS which also enables us to evaluate the SNR performance. First, we consider a case that a source antenna serves as a point source located at $\mathbf{r}$; this assumption is reasonable when the source is located in the farfield region, and the amplitude of the spherical waves $\Phi(\mathbf{r})$ emitted from the source to the IRS, which is located at $\mathbf{r}^{\prime}$, and it is denoted as $\Phi\left(\mathbf{r}^{\prime} \mid \mathbf{r}\right)$. Now, $\Phi\left(\mathbf{r}^{\prime} \mid \mathbf{r}\right)$ is the solution of Helmholtz equation

$$
\nabla^{2} \Phi\left(\mathbf{r}^{\prime} \mid \mathbf{r}\right)+k^{2} \Phi\left(\mathbf{r}^{\prime} \mid \mathbf{r}\right)=-\delta\left(\mathbf{r}^{\prime}-\mathbf{r}\right),
$$

where $\nabla$ denotes the gradient of a vector field and $\delta\left(\mathbf{r}^{\prime}-\mathbf{r}\right)$ is the three dimensional Dirac delta function. Thus, we obtain the solution as a scalar Green's function:

$$
\Phi\left(\mathbf{r}^{\prime} \mid \mathbf{r}\right)=\frac{e^{j k\left|\mathbf{r}^{\prime}-\mathbf{r}\right|}}{4 \pi\left|\mathbf{r}^{\prime}-\mathbf{r}\right|}=\frac{e^{j k R}}{4 \pi R},
$$

where $k$ is the wave number in the free space, and defined as $k=\frac{2 \pi}{\lambda}$. The electrical field strength, $E_{\mathrm{s}}$, captured by a rectangular aperture from a point source, can be expressed in Cartesian coordinates as

$$
E_{\mathrm{s}}=\int_{-\frac{a}{2}}^{\frac{a}{2}} \int_{-\frac{b}{2}}^{\frac{b}{2}} \frac{e^{j k R}}{4 \pi R} e^{-j k\left(\mathbf{r}^{\prime} \cdot \hat{\mathbf{r}}\right)} d x d y
$$

where $\mathbf{r}^{\prime}=x \hat{\mathbf{x}}+y \hat{\mathbf{x}}$ and $\hat{\mathbf{r}}=\sin \theta \cos \phi \hat{\mathbf{x}}+\sin \theta \sin \phi \hat{\mathbf{y}}$. As a result, (27) becomes:

$$
E_{\mathrm{s}}=\frac{e^{j k R}}{4 \pi R} \int_{-\frac{a}{2}}^{\frac{a}{2}} \int_{-\frac{b}{2}}^{\frac{b}{2}} e^{-j k \sin \theta(\cos \phi x+\sin \phi y)} d x d y .
$$

As the radiated power per unit area is proportional to the square of the electric field strength, the power pattern of the spherical wavefronts (28) reaching the rectangular aperture is

$$
\begin{aligned}
& A(\theta, \phi) \\
& \quad=\left(\frac{e^{j k R} a b \operatorname{sinc}\left(\frac{k a}{2} \sin \theta \cos \phi\right) \operatorname{sinc}\left(\frac{k b}{2} \sin \theta \sin \phi\right)}{4 \pi R}\right)^{2},
\end{aligned}
$$

where $\operatorname{sinc}(x)=\frac{\sin (x)}{x}$.

Now, we introduce the tapering level, which measures how much the electrical field is tapered at the aperture edge $\left(A_{\text {edge }}\right)$ 
when compared to the centre of the IRS $\left(A_{\text {centre }}\right)$. From this definition, we can express the tapering level as

$$
T L=\frac{A(\theta, \phi)}{A(0,0)}=\frac{A_{\text {edge }}}{A_{\text {centre }}} .
$$

By setting the tapering level to a certain value, we can determine the corresponding size of the reflecting array, spillover and taper efficiency. Table I shows how these different parameters behave under different carrier frequencies $\left(f_{\mathrm{c}}\right)$, where $R$ is the far-field distance $\left(R=2 \max \left\{a^{2}, b^{2}\right\} / \lambda\right)$ and $T L=-20 \mathrm{~dB}$, leading to different efficiencies. When the operating frequency is increased, the size of IRS can be reduced to maintain the same level of loss from the center toward the edge of the reflecting area. In this case, the far-field definition becomes more relevant when radiation from the IRS is considered. This is because when we consider the radiated field from the IRS aperture (under the calculated spillover and taper efficiencies), the IRS becomes the source of radiation for SNR calculations.

TABLE I: Spillover and taper efficiency with tapering level $-20 \mathrm{~dB}$ at different frequencies.

\begin{tabular}{c|c|c|c}
\hline$f_{\mathrm{c}}(\mathrm{GHz})$ & IRS diameter $(\mathrm{m})$ & $\varepsilon_{\mathrm{s}}$ & $\varepsilon_{\mathrm{t}}$ \\
\hline 3 & 0.072 & 0.4598 & 0.8933 \\
5 & 0.043 & 0.1756 & 0.9751 \\
28 & 0.02 & 0.0059 & 0.993 \\
\hline
\end{tabular}

After elaborating on the diameter of the IRS, we can finally calculate the signal-to-noise ratio (SNR) with respect to the location of source, IRS and destination. We note that in [10], the SNR was defined as

$$
\mathrm{SNR}=\frac{\left(\sqrt{\beta_{\mathrm{IRS}}}+\sqrt{\beta_{\mathrm{SD}}}\right)^{2} P_{\mathrm{S}}}{\sigma^{2}},
$$

where $\beta_{\mathrm{SD}}=\frac{G_{\mathrm{S}} G_{\mathrm{D}} \lambda^{2}}{\left(4 \pi R_{\mathrm{S}, \mathrm{D}}\right)^{2}}$, the distance between the source and destination is denoted as $R_{\mathrm{S}, \mathrm{D}}$, and $\sigma^{2}$ denotes the noise power. Figure 3 shows the SNR performance for the proposed model and the model considered in [10]: we choose $R_{\mathrm{S}, \mathrm{D}}=5 \mathrm{~m}$, $T L=-20 \mathrm{~dB}, P_{\mathrm{S}} / \sigma^{2}=5 \mathrm{~dB}, G_{\mathrm{S}}=G_{\mathrm{D}}=5 \mathrm{~dB}$ and $f_{\mathrm{c}}=3 \mathrm{GHz}$, while the location of IRS is moving from the source towards the destination. It is observed from the figure that the performance predicted in [10] overestimates the achievable SNR compared to our model, that takes into account the aperture efficiency. Moreover, when $R_{\mathrm{S}, \mathrm{IRS}}$ and $\theta_{\text {axis }}$ increase, the power captured by the IRS and the aperture efficiency decrease; this leads to even lower receiving power at the destination; thus, the SNR should be a non-increasing function with respect to $R_{\mathrm{S} \text {,IRS }}$ and $\theta_{\text {axis }}$, and it can be seen that our expression is able to capture this phenomenon, while the expression in [10] does not consider the affect of aperture efficiency and leads to slight overestimation of the SNR as the IRS approaches to the destination.

\section{CONCLUSION}

We studied an important parameter in designing an IRSbased communication system, i.e. aperture efficiency. We introduced two major contributions to the aperture efficiency concept, which are the spillover efficiency and the taper

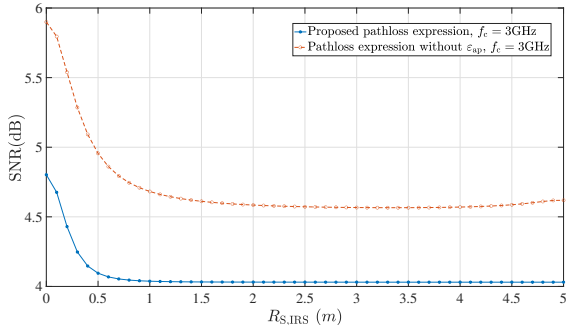

Fig. 3: SNR comparison between the proposed model and [10].

efficiency, and presented that improving the efficiency can enable us to further boost the pathloss performance. When it comes to a real-world deployment of an IRS and because the size of the aperture is always a concern, we also studied the aperture size and proposed a practical way to derive the IRS diameter. Our results demonstrated that there is a tradeoff between the distance from the source to the IRS and the diameter of the aperture size due to the far-field constraint. Finally, we proposed a pathloss expression in the far-field zone, which is connected with the tilt angle and how much of the electrical field is captured and reflected by an IRS.

\section{REFERENCES}

[1] H. Q. Ngo, A. Ashikhmin, H. Yang, E. G. Larsson, and T. L. Marzetta, "Cell-free massive MIMO versus small cells," IEEE Trans. Wireless Commun., vol. 16, no. 3, pp. 1834-1850, Mar. 2017.

[2] M. Xiao et al., "Millimeter wave communications for future mobile networks," IEEE J. Sel. Areas Commun., vol. 35, no. 9, pp. 1909-1935, Sep. 2017.

[3] C. Liaskos et al., "A new wireless communication paradigm through software-controlled metasurfaces," IEEE Commun. Mag., vol. 56, no. 9, pp. 162-169, Sep. 2018.

[4] M. Matthaiou et al., "The road to 6G: Ten physical layer challenges for communications engineers,", IEEE Commun. Mag., 2020.

[5] T. Nakanishi, T. Otani, Y. Tamayama, and M. Kitano, "Storage of electromagnetic waves in a metamaterial that mimics electromagnetically induced transparency," Physical Review B, vol. 87, no. 161110, 2013.

[6] A. Silva et al., "Performing mathematical operations with metamaterials," Science, vol. 343, no. 6167, pp. 160-163.

[7] Q. Wu and R. Zhang, "Weighted sum power maximization for intelligent reflecting surface aided SWIPT," IEEE Wireless Commun. Lett., vol. 9, no. 5, pp. 586-590, May 2020.

[8] X. Yue and Y. Liu, "Performance analysis of intelligent reflecting surface assisted NOMA networks," 2020.

[9] M. Cui, G. Zhang, and R. Zhang, "Secure wireless communication via intelligent reflecting surface," IEEE Wireless Commun. Lett., vol. 8, no. 5, pp. 1410-1414, Oct. 2019.

[10] O. Özdogan, E. Björnson, and E. G. Larsson, "Intelligent reflecting surfaces: Physics, propagation, and pathloss modeling," IEEE Wireless Commun. Lett., vol. 9, no. 5, pp. 581-585, May 2020.

[11] C. Pan et al., "Multicell MIMO communications relying on intelligent reflecting surfaces," IEEE Trans. Wireless Commun., vol. 19, no. 8, pp. 5218-5233, 2020.

[12] G. Zhou, C. Pan, H. Ren, K. Wang, and A. Nallanathan, "Intelligent reflecting surface aided multigroup multicast MISO communication systems," IEEE Trans. Signal Process., vol. 68, p. 3236-3251, Apr. 2020.

[13] C. A. Balanis, Antenna Theory: Analysis and Design. USA: WileyInterscience, 2005.

[14] A. Z. Elsherbeni, F. Yang, and P. Nayeri, Reflectarray Antennas: Theory, Designs, and Applications. John Wiley and Sons, 2018.

[15] Q. Wu and R. Zhang, "Towards smart and reconfigurable environment: Intelligent reflecting surface aided wireless network," IEEE Commun. Mag., vol. 58, no. 1, pp. 106-112, Jan. 2020.

[16] D. R. Smith et al., "An analysis of beamed wireless power transfer in the Fresnel zone using a dynamic, metasurface aperture," Journal of Applied Physics, vol. 121, no. 1, p. 014901, Jan. 2017. 